\title{
EFEITO AGUDO DO LASER DE BAIXA POTÊNCIA NA FADIGA DO BÍCEPS BRAQUIAL DE ATLETAS DE VOLEIBOL
}

\author{
ACUTE EFFECT OF LOW-POWER LASER IN FATIGUE OF BICEPS BRACHII OF VOLLEYBALL ATHLETES
}

EFECTO AGUDO DEL LÁSER DE BAJA POTENCIA EN LA FATIGA DEL BÍCEPS BRAQUIAL

Artigo Original

Original ARTICLE

DEATLETASDE VOLEIBOL

Juliana Lôbo Froio ${ }^{1}$

(Fisioterapeuta)

Ana Claudia Muniz Renno²

(Fisioterapeuta)

Eduardo Federighi Baisi Chagas ${ }^{3}$ (Profissional de Educação Física)

Felipe Guilherme Leite de Campos (Fisioterapeuta)

Renata Lumena Altruda Pucci ${ }^{1}$

(Fisioterapeuta)

Marcos Seizo Kishi ${ }^{4}$

(Fisioterapeuta)

Cristiane Rodrigues Pedroni'

(Fisioterapeuta)

1. Universidade Estadual Paulista Júlio de Mesquita Filho - UNESP, Departamento de Fisioterapia e Terapia Ocupacional, Marília, São Paulo, SP, Brasil.

2. Universidade Federal de São

Paulo - UNIFESP, Departamento de Biociências, Santos, São Paulo,

SP, Brasil.

3. Universidade de Marília -

UNIMAR, Marília, São Paulo,

SP, Brasil.

4. Universidade Federal de

Uberlândia - UFU, Faculdade de

Educação Física e Fisioterapia,

Uberlândia, Minas Gerais, MG, Brasil.

\section{Correspondência:}

Cristiane Rodrigues Pedroni Universidade Estadual Paulista Júlio de Mesquita Filho. Av. Hygino Muzzi Filho, 737, Bairro Mirante, Marília, São Paulo, SP, Brasil. 17525-000. pedronicr@marilia.unesp.br

\section{RESUMO}

Introdução: A laserterapia de baixa potência tem entre seus propósitos auxiliar a recuperação de tecidos biológicos, atenuando os efeitos da fadiga muscular e contribuindo com a melhora do desempenho em atletas. Aspectos metodológicos ainda limitam as conclusões do efeito agudo da laserterapia sobre o desempenho muscular. Objetivo: Verificar o efeito agudo do laser terapêutico de baixa potência na fadiga induzida do bíceps braquial de atletas de voleibol. Métodos: Este foi um estudo randomizado e duplo-cego, aprovado pelo Comitê de Ética da UNESP de Marília. Participaram do estudo 19 atletas de voleibol de ambos os sexos. Realizou-se coleta de dados eletromiográficos do músculo bíceps braquial no exercício isométrico de flexão de cotovelo antes e após a aplicação do laser terapêutico. Um haltere com 75\% do pico de força, obtido por uma célula de carga, foi utilizado para o protocolo de fadiga. Em seguida, os voluntários foram submetidos à aplicação do laser (ativo ou placebo) em seis pontos do músculo bíceps braquial. Os dados eletromiográficos foram analisados no domínio da frequência, utilizando-se o software Myosystem ${ }^{\circledR}$. Verificou-se a distribuição de normalidade dos dados pelo teste de Shapiro-Wilk, e utilizou-se Anova (split plot) de medidas repetidas a fim de testar a interação entre tempo e grupo. Resultados: Para nenhuma das variáveis analisadas foi observada interação significativa entre grupo e tempo, indicando que o grupo irradiado não apresentou vantagens com relação ao grupo placebo. Conclusão: Após o protocolo de fadiga proposto, uma única aplicação de laser de baixa potência não foi suficiente para produzir efeitos positivos no desempenho de força e no sinal eletromiográfico do músculo bíceps braquial de atletas de voleibol.

Descritores: fototerapia; eletromiografia; fadiga muscular; voleibol.

\section{ABSTRACT}

Introduction: Low-power laser therapy has among its aims to aid the recovery of biological tissues, attenuating the effects of muscle fatigue and contributing to the improvement of performance in athletes. Methodological aspects still limit the conclusions of the acute effect of laser therapy on muscular performance. Objective: To verify the acute effect of low-power therapeutic laser in the induced fatigue in biceps brachii of volleyball athletes. Methods: This was a randomized double-blind study, approved by Institutional Review Board of UNESP, Marilia. Nineteen volleyball athletes of both sexes participated in this study. Electromyographic data were collected from the biceps brachii muscle in isometric exercise of the elbow flexion before and after the application of the therapeutic laser. A dumbbell with 75\% of the force peak, obtained by a load cell, was used for the fatigue protocol. The volunteers then underwent laser (active or placebo) application on six points of the biceps brachii muscle. The EMG data were analyzed in the frequency domain using the Myosystem ${ }^{\circledR}$ software. The distribution of normality was verified by the Shapiro-Wilk test, and repeated measures ANOVA (split plot) was used to test the interaction between time and group. Results: No significant interaction between group and time was observed for any analyzes variables, indicating that the irradiated group did not show advantages over the placebo group. Conclusion: After the proposed fatigue protocol, a single low-power laser application was not sufficient to produce positive effects on strength performance and on the electromyographic signal of the biceps brachii muscle of volleyball athletes.

Keywords: phototherapy; electromyography; muscle fatigue; volleyball.

\section{RESUMEN}

Introducción: La láserterapia de baja potencia tiene entre sus propósitos auxiliar la recuperación de los tejidos biológicos, atenuando los efectos de la fatiga muscular y contribuyendo con la mejora del desempeño en atletas. Los aspectos metodológicos todavía limitan las conclusiones del efecto agudo de la láserterapia sobre el rendimiento muscular. Objetivo: Verificar el efecto agudo del láser terapéutico de baja potencia en la fatiga inducida del bíceps braquial de atletas de voleibol. Métodos: Fue un estudio aleatorizado y doble ciego, aprobado por el Comité de Ética de la UNESP de Marília. Participaron del estudio 19 atletas de voleibol de ambos sexos. Se realizó la recolección de datos electromiográficos del músculo bíceps braquial en ejercicio isométrico de flexión de codo antes y después de la aplicación del láser terapéutico. Se utilizó una mancuerna con un 75\% del pico de fuerza, obtenido por una célula de carga para el protocolo de fatiga. A continuación, los voluntarios fueron sometidos a la aplicación del láser 
(activo o placebo) en seis puntos del músculo bíceps braquial. Los datos electromiográficos se analizaron en el ámbito de la frecuencia, utilizando el software Myosystem ${ }^{\circledR}$. Se verificó la distribución de la normalidad de los datos por la prueba de Shapiro-Wilk, y se utilizó Anova (split plot) de medidas repetidas para probar la interacción entre tiempo y grupo. Resultados: Para ninguna de las variables analizadas se observó interacción significativa entre grupo y tiempo, indicando que el grupo irradiado no presentó ventajas con relación al grupo placebo. Conclusión: Después del protocolo de fatiga propuesto, una única aplicación láser de baja potencia no fue suficiente para producir efectos positivos en el desempeño de fuerza y en las señales electromiográficas del músculo bíceps braquial de atletas de voleibol.

\section{Descriptores: fototerapia; electromiografía; fatiga muscular; voleibol.}

\section{INTRODUÇÃO}

O voleibol mesmo em categorias juvenis é caracterizado pelo alto volume de ações motoras, principalmente durante as sessões de treino, o que representa uma sobrecarga mecânica e metabólica excessiva, evidenciada pela redução da capacidade coordenativa e de produção de força, que se associa fortemente a ocorrência de lesões esportivas ${ }^{1,2}$, em especial nos membros superiores, devido a alta exigência mecânica decorrente dos fundamentos de bloqueio e ataque do voleibol ${ }^{3}$.

Muitos eventos englobam o início da atividade muscular voluntária, começando com o controle cortical no cérebro e terminando com a formação das pontes cruzadas dentro da fibra muscular. Se ocorrer falha de qualquer um dos processos envolvidos na contração muscular, é possível que seja iniciado o processo da fadiga muscular ${ }^{4}$, que consiste na incapacidade da musculatura em manter uma produção de força esperada ${ }^{5}$.

Neste âmbito, vários estudos têm buscado o desenvolvimento de técnicas visando a prevenção ou redução dos efeitos da fadiga, especialmente em atletas ${ }^{1,6,7}$. Entre esses recursos, a Laserterapia de Baixa Potência (LBP) eclodiu como um instrumento crucial capaz de interagir nos tecidos biológicos, produzindo vários efeitos fisiológicos e/ou terapêuticos, dentre eles a melhora do desempenho do músculo estriado. Este método consiste na aplicação terapêutica de diodos superluminosos monocromáticos de intensidade relativamente baixa para que não efetuem qualquer aquecimento detectável dos tecidos irradiados, visando o tratamento de afecções e lesões ${ }^{8,9}$.

Ferraresi e colaboradores ${ }^{10}$ perceberam que, no momento em que as células são irradiadas com várias faixas de luz, esta luz é absorvida pela cadeia respiratória. Os primeiros episódios fotoquímicos e fotofísicos sucedem na mitocôndria e na membrana citoplasmática. A mitocôndria possui um mecanismo para a reabsorção de oxigênio e este tem potencial para ser uma fonte de elétrons para a fosforilização oxidativa de ATP sob as condições fisiológicas.

Neste mesmo estudo verificou-se que a melhoria do desempenho muscular após a irradiação do laser pode estar relacionada à remoção e oxidação do ácido láctico, produto do metabolismo anaeróbico durante o exercício e que transforma-se em lactato para ser armazenado nos músculos; de modo que, uma vez aplicado o LBP, há a redução do lactato concentrado e uma maior disponibilidade da quantidade de energia durante o exercício ${ }^{10}$.

O presente estudo tem como objetivo verificar o efeito agudo do laser terapêutico de baixa potência na fadiga induzida do bíceps braquial de atletas de voleibol. A hipótese deste estudo é a de que uma única aplicação de laser seria capaz de retardar o processo de fadiga e, por conseguinte, tanto a perda de força quanto a redução do valor da frequência mediana do sinal eletromiográfico seriam menores no grupo irradiado.

\section{MATERIAIS E MÉTODOS}

A coleta de dados foi realizada após aprovação pelo Comitê de Ética em Pesquisa da Faculdade de Filosofia e Ciências da Universidade
Estadual Paulista Júlio de Mesquita Filho - UNESP, Marília" e de acordo com a Resolução 466/12 do Conselho Nacional de Saúde sob o protocolo de 2014-1061.

Participaram desta pesquisa 19 atletas de voleibol masculino e feminino, com idade entre 15 e 22 anos. Todos os sujeitos foram informados sobre a proposta e os procedimentos deste estudo, e inicialmente responderam um questionário com dados gerais, antropométricos e sobre as lesões sofridas na atual temporada e assinaram um Termo de Consentimento Livre e Esclarecido.

Considerou-se como critérios de elegibilidade sujeitos que eram praticantes de voleibol e que não sofreram lesão muscular no membro superior dominante nos dois meses anteriores à realização da pesquisa.

Esta pesquisa foi constituída de um estudo duplo-cego e randomizado. Assim, após a seleção dos sujeitos, foi realizado um sorteio para definir qual o procedimento de aplicação do laser o atleta iria receber, formando dois grupos:

a. Sujeitos que receberam aplicação de laser ativo $(n=10) ; e_{\text {, }}$

b. Sujeitos que receberam aplicação de laser placebo $(n=9)$.

Para avaliar o pico de força isométrica do músculo bíceps braquial, foi realizada uma Contração Isométrica Voluntária Máxima (CIVM) de cinco segundos para familiarização do procedimento, e uma CIVM de 15 segundos, sendo respeitado um intervalo de dois minutos entre cada CIVM. Utilizou-se de uma estação de testes isométricos que permitiu a realização destas contrações para a flexão do cotovelo dominante. Para isso, os voluntários permaneceram em posição ortostática, tronco ereto, cotovelo a $90^{\circ}$ e braço estabilizado junto ao tronco. Uma alça de ferro foi colocada na mão dominante dos voluntários para a realização da força de tração na célula de carga (EMG System do Brasil ${ }^{\circledR}$, software AqDados com resolução em 1 kgf e sensibilidade de 0,1 kgf) que enviava as informações adquiridas a um computador. Os voluntários foram orientados previamente sobre a execução do exercício, bem como estimulados com comando verbal a não permitir o uso de compensações biomecânicas que favorecessem o aumento do valor da força. O valor de pico de força isométrica máxima foi determinado pela CIVM que gerou o maior valor de força de tração da célula de carga.

Simultaneamente ao teste isométrico, foram coletados sinais eletromiográficos do músculo bíceps braquial, utilizando um módulo de aquisição de sinais biológicos Modelo MyosystemBr1_P84 (Data Hominis ${ }^{\circledR}$ ) de 8 canais, calibrado com frequência de amostragem de $2000 \mathrm{~Hz}$, ganho total de 2000 vezes (20 vezes no sensor e 100 vezes no equipamento). A análise do sinal EMG foi realizada em rotinas específicas disponíveis no software do eletromiógrafo, por meio do valor de Frequência Mediana por meio da transformada rápida de Fourier (FFT). Para analisar o comportamento da frequência mediana ao longo do tempo, o sinal eletromiográfico foi dividido em três janelas divididas em cinco segundos cada.

Utilizou-se filtro passa-alta de $20 \mathrm{~Hz}$, passa-baixa de $1000 \mathrm{~Hz}$. Foi utilizado eletrodo de superfície de Ag/AgCl (Data Hominis ${ }^{\circledR}$ ), em configuração bipolar, com área de captação de $1 \mathrm{~cm}$ de diâmetro. 
Para a execução da coleta, o eletrodo foi colocados $1 / 3$ a partir da fossa cubital na linha entre o acrômio e fossa cubital, de acordo com orientações da "Surface EMG for Non Invasive Assessment of Muscles" (SENIAM) ${ }^{11}$. Um eletrodo circular foi posicionado sobre o epicôndilo medial do membro ipsilateral como um eletrodo de referência para reduzir a aquisição de ruídos. Primeiramente limpou-se com álcool a área da pele em que foram colocados os eletrodos e, quando necessário, tricotomia da região, realizados para a redução da impedância da pele e melhor fixação dos eletrodos.

Após esta etapa eletromiográfica de contração isométrica, os sujeitos foram submetidos a um protocolo de fadiga do músculo bíceps braquial, que se resumiu no maior número de flexões de cotovelo possíveis realizados pelo lado dominante, no tempo máximo conseguido sem compensações biomecânicas até a exaustão, com carga determinada por 75\% da carga máxima obtida com a célula de carga, conforme realizado em estudo de Leal-Junior ${ }^{6}$.

A laserterapia foi realizada imediatamente após a execução do protocolo de fadiga. Foi utilizado neste estudo o Laser AsGaAl (808nm; Photon Lase III; DMC ${ }^{\circledR}$, São Carlos, SP, Brasil). Os parâmetros utilizados foram: potência de $100 \mathrm{~mW}$, energia por ponto igual a $7 \mathrm{~J}$, densidade de energia de 250J/ $\mathrm{cm}^{2}$, energia total de $56 \mathrm{~J}$ e tempo de irradiação igual a um minuto e 10 segundos por ponto. A aplicação foi feita de modo pontual, em contato com a pele do sujeito, em seis pontos distribuídos uniformemente no ventre do músculo bíceps braquial do membro dominante. A caneta do laser foi fixada a um ângulo de $90^{\circ} \mathrm{em}$ cada um dos seis pontos de aplicação. Para o grupo placebo, a mesma metodologia foi adotada, porém sem ligar o aparelho.

Na sequência, repetiram-se os testes realizados no dinamômetro isométrico para que fosse avaliado o efeito agudo da aplicação do laser sobre a atividade muscular após a fadiga.

Os resultados foram organizados sob a forma de estatística descritiva, com valores de média e desvio padrão. A distribuição de normalidade foi verificada pelo teste de Shapiro-wilk com correção de Lillifors. Para analisar as diferenças entre os grupos placebo e irradiado no momento pré-protocolo de fadiga foi aplicado o teste $t$ Student para amostras independentes. Para analisar as diferenças entre os momentos pré e pós-protocolo de fadiga dentro de cada grupo foi aplicado o teste $t$ Student para amostras pareadas. Foi construída uma Anova (Split Plot) para medidas repetidas com o objetivo de testar a interação entre tempo (momento pré e pós-protocolo de fadiga) e grupo (irradiado e placebo). No caso de rejeição da hipótese de esfericidade, as análises foram baseadas no teste multivariado de Greenhouse-Geisser, Quando o efeito do tempo foi estatisticamente significante foi realizado o teste Post Hoc de Fishers Least Significant Difference (LSD) para localizar as diferenças. Para todas as análises utilizou-se o software SPSS versão 19.0 for windows, sendo adotado nível de significância de 5\%. Para todas as análises utilizou-se o software SPSS versão 19.0 for windows, sendo adotado nível de significância de 5\%.

\section{RESULTADOS}

A Tabela 1 apresenta a comparação entre os grupos placebo e irradiado para a variável idade e índice de massa corporal (IMC). Não foram observadas diferenças significativas entre os grupos quanto à idade e o IMC, sugerindo que estas podem não ter se associado ao comportamento das variáveis de interesse entre os grupos.

A Tabela 2 apresenta os resultados da comparação entre os grupos placebo e irradiado para os momentos pré e pós-protocolo de fadiga. Quanto aos valores de pico de força (PF15s), ambos os grupos demonstraram redução significativa entre os momentos pré e pós-protocolo de fadiga. Quando avaliados os valores de frequência mediana (FM15s), não foram observadas diferenças significativas tanto no grupo placebo quanto no irradiado.
Tabela 1. Comparação entre os grupos para as variáveis idade e IMC.

\begin{tabular}{c|c|c|c}
\hline \multicolumn{3}{|c|}{ Grupos } & \\
\hline & Placebo $(\mathbf{n}=\mathbf{9})$ & Irradiado $(\mathbf{n = 1 0})$ & $\mathbf{P}$ \\
\cline { 2 - 4 } & Média $\pm \mathbf{D P}$ & Média $\pm \mathbf{D P}$ & \\
\hline Idade & $17,4 \pm 2,5$ & $17,1 \pm 2,2$ & 0,758 \\
\hline IMC & $21,8 \pm 1,3$ & $19,7 \pm 0,9$ & 0,156 \\
\hline
\end{tabular}

Nota: * $p \leq 0,05$ diferenças significativas entre os grupos. Teste $t$ Student.

Tabela 2. Comparação entre os grupos placebo e irradiado para os momentos pré e pós-protocolo de fadiga.

\begin{tabular}{|c|c|c|c|c|c|c|c|}
\hline & \multicolumn{6}{|c|}{ Grupos } & \multirow[b]{3}{*}{ Interação } \\
\hline & \multicolumn{3}{|c|}{ Placebo $(n=9)$} & \multicolumn{3}{|c|}{ Irradiado $(n=11)$} & \\
\hline & Pré & Pós & & Pré & Pós & & \\
\hline & $M \pm D P$ & $M \pm D P$ & $p$ & $M \pm D P$ & $M \pm D P$ & $p$ & $p$ \\
\hline FM 15s & $55,6 \pm 9,2$ & $63,0 \pm 16,9$ & 0,095 & $62,0 \pm 10,5$ & $62,8 \pm 13,2$ & 0,808 & 0,190 \\
\hline PF 15s & $8,5 \pm 2,0$ & $6,6 \pm 1,4$ & $0,001^{*}$ & $8,0 \pm 2,4$ & $6,0 \pm 2,8$ & $0,0001^{*}$ & 0,840 \\
\hline
\end{tabular}

A Figura 1 (Box Plot) apresenta o comportamento das variáveis FM para os momentos pré e pós-protocolo de fadiga entre os grupos placebo e irradiado para as Janelas de tempo 1, 2 e 3. Não foi detectado interação significativa entre grupo e tempo (janelas) para as variáveis FMpré e FMpós.

Entretanto, os valores de FM apresentaram variações significativas entre as janelas dentro dos grupos (Figura 2), tanto no momento pré, quanto pós-protocolo de fadiga. Para o grupo placebo foi observado diferenças significantes nos valores de FM entre as janelas 1 e $3(p=0,025)$ no momento pré, e entre as janelas 1 e $3(p=0,015)$ no momento pós. No grupo irradiado, a FM no momento pré apresentou diferenças significantes entre as janelas 1 e $3(p=0,002)$ e entre as janelas 2 e $3(p=0,044)$, sugerindo que o grupo irradiado apresentou menor resistência à fadiga no momento pré-protocolo. No momento pós-protocolo de fadiga o grupo irradiado apresentou diferenças significantes nos valores de FM entre as 1 e $2(p=0,006)$ e entre as janelas 1 e $3(p=0,0001)$.

Ao observar a variação delta entre as janelas 2 e 1 (pré), janelas 3 e 1 (pré), janelas 2 e 1 (pós) e janelas 3 e 1 (pós), fica claro que ambos os grupos apresentaram redução da FM em relação aos valores iniciais verificados na janela 1, tanto no momento pré, quanto pós protocolo de fadiga.

Apesar da reta de regressão do grupo placebo (Figura 3) apresentar um inclinação positiva, esta apenas indica que as reduções foram de menor amplitude, mas estiveram presente.

Diferente do grupo placebo, o grupo irradiado, tanto no momento pré, quanto pós-protocolo de fadiga, apresentou um $\mathrm{R}^{2}$ significativo indicando que no grupo irradiado os maiores valores observados na janela 1 se correlacionaram a maiores reduções da FM observadas nas variações delta entre as janelas 2 e 1, e janelas 3 e 1.

Se de fato os valores mais elevados da FM na janela 1 se relacionam com maior redução da FM para as janelas 2 e 3, é possível que os valores de FM na janela 1 do grupo placebo inferiores aos do grupo irradiado tenham sido um aspectos importante para não se observar diferenças entre os grupos.

Lembro ainda, que em protocolos de fadiga a motivação e empenho individual para a produção de níveis máximos de força, são fatores de grande influência sobre a observação da fadiga, pois, caso no momento pré-protocolo o fadiga o avaliado não desempenhe seu máximo existe uma tendência deste produzir desempenhos melhores após o protocolo de fadiga.

De qualquer forma, os resultados apresentados nas Figuras 2 e 3, indicam que diferenças de desempenho entre os grupos no momento pré- intervenção podem representar um forte fator de influência e levar a conclusões equivocadas sobre os reais efeitos da LBP. 


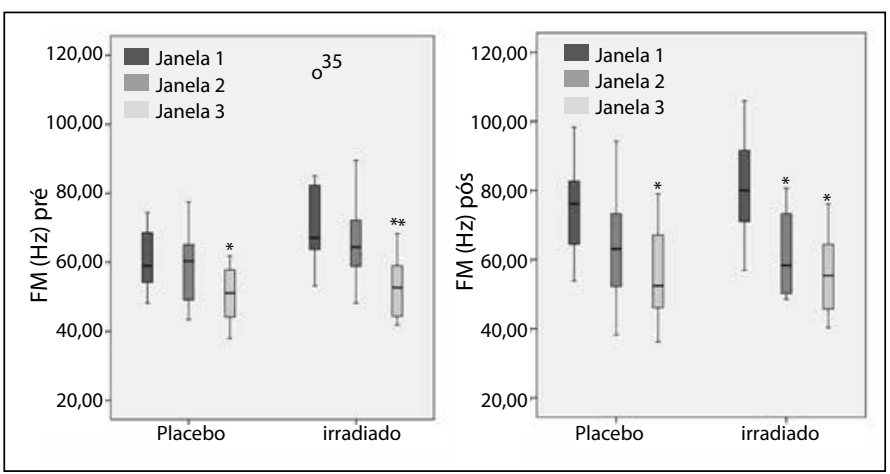

Figure 1. Comportamento da FM para o grupo irradiado e placebo nos momentos pré e pós-protocolo de fadiga, durante as CIVMs de 15 segundos, onde o sinal eletromiográfico foi dividido em três janelas de 5 segundos (janela 1 (azul), 2 (verde) e 3 (marrom)).

* diferença em relação a janela 1 dentro do grupo. $¥$ diferença em relação a janela 2 dentro do grupo.

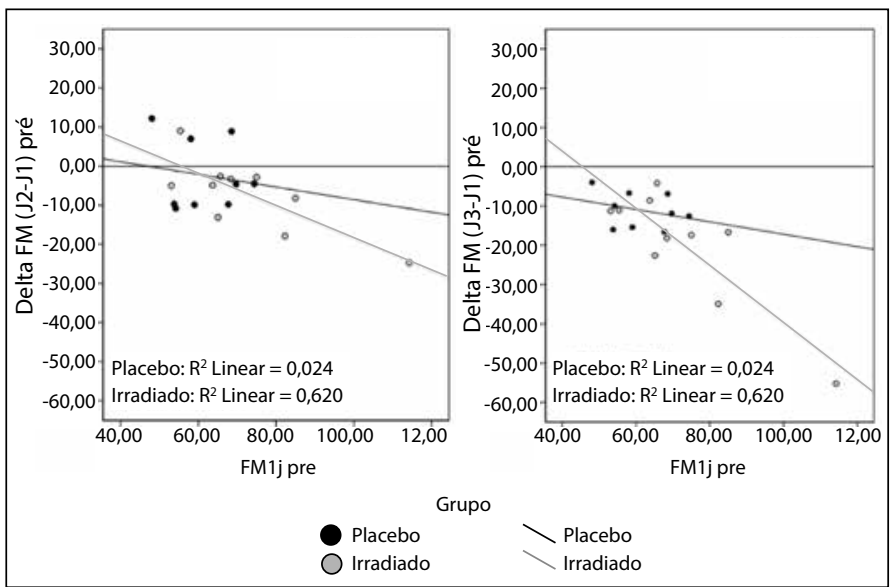

Figure 2. Análise da variação delta entre as janelas 2 e 1, e janelas 3 e 1 em relação aos valores iniciais da janela para os grupos placebo (preto) e irradiado (cinza) no momento pré-protocolo de fadiga.

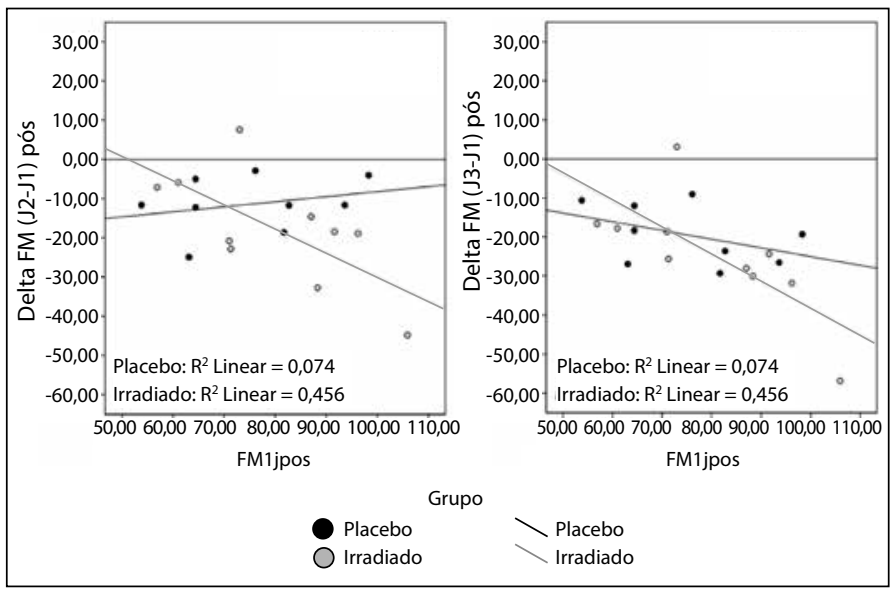

Figure 3. Análise da variação delta entre as janelas 2 e 1, e janelas 3 e 1 em relação aos valores iniciais da janela para os grupos placebo (preto) e irradiado (cinza) no momento pré-protocolo de fadiga.

\section{DISCUSSÃO}

De acordo com os resultados obtidos no presente estudo, não foram encontradas diferenças significativas na atividade eletromiográfica (variável da FM) e força do músculo bíceps braquial após uma única aplicação do laser de baixa potência, posteriormente ao protocolo de fadiga muscular.

A fadiga muscular é um processo multifatorial que envolve danos fisiológicos e biomecânicos, recorrentes de uma utilização exacerbada de um determinado músculo ou grupo muscular ${ }^{12}$. Alguns fatores podem afetar a produção de força durante atividade muscular como: o tipo e intensidade de exercício, os grupos musculares envolvidos nos movimentos realizados e o ambiente bioquímico muscular ${ }^{13,14}$. Idade e gênero também são importantes fatores para determinar a habilidade de contração muscular e resistência à fadiga ${ }^{14}$. Sintetizando, fadiga muscular é um fenômeno complexo que envolve elementos fisiológicos, biomecânicos e psicológicos ${ }^{12}$

Considerada fator que predispõe a ocorrência de lesões ${ }^{5,15}$, a fadiga tem como característica o fato de ser dependente da tarefa, isto é, possuir causas e comportamento de acordo com a forma pela qual a mesma é induzida ${ }^{16}$. Deste modo, talvez a maneira que a fadiga foi induzida neste estudo não tenha atingido os níveis de fadiga necessários para promoção do efeito da laserterapia, e outros estudos deveriam estudar o comportamento da aplicação do LBP por meio de indução de fadiga por outros procedimentos.

Estudos apontam os mecanismos geradores de lesões no voleibol. Santos et al. ${ }^{17}$ assinalaram que o bloqueio e o ataque representaram mais do que o dobro dos outros mecanismos de lesão, devido à elevada demanda no membro superior dominante, gerando maior desgaste das estruturas musculares. De tal análise percebe-se que o tempo detectado entre os impactos pode não estar sendo suficiente para o reparo de biomateriais, resultando em sobrecargas significativas e no acometimento de lesões, e é nesse momento do exercício que a aplicação do laser pode surgir de forma crucial. No presente estudo realizou-se a aplicação no músculo bíceps braquial por agir tanto excêntrica quanto concentricamente em tais movimentos apontados como os mais lesivos na modalidade esportiva.

Alguns estudos apontaram que o método apresentou-se de modo positivo no desempenho muscular de atletas. Um deles, realizado com atletas de voleibol, envolveu a aplicação de LBP no bíceps braquial do membro superior dominante no momento anterior ao protocolo de fadiga e sua avaliação consistia na medição de marcadores bioquímicos e número de repetições de flexo-extensão de cotovelo; concluiu-se que houve um aumento significativo do número de repetições em comparação ao grupo placebo ${ }^{6}$. Ao contrário deste trabalho, o presente estudo realizou a aplicação da LBP após o protocolo de fadiga, o que pode deduzir a possível diferença dos resultados.

Estudos com LBP realizados em outro grupo muscular, o quadríceps, verificaram melhora do método sobre o desempenho muscular. No primeiro, a aplicação ocorreu em grupos pré e pós-protocolo de fadiga, e percebeu-se que houve melhora do desempenho dos atletas submetidos à aplicação tanto antes quanto após, com maior benefício nos atletas submetidos à aplicação pós, além de apontar que o laser foi efetivo na remoção da concentração de marcadores bioquímicos da fadiga ${ }^{18}$. Ferraresi et al. ${ }^{10}$ aplicaram LPB bilateralmente após um protocolo de fadiga diferente do anterior, consistido em 50 repetições divididas em cinco séries com o aparelho "leg press", realizado 12 semanas consecutivas, e indicou-se que o laser foi eficaz para potencializar o desempenho muscular em comparação com apenas o treinamento de força. Este último estudo é de conveniente atenção, visto que trata-se da aplicação de modo crônico; sendo de valia notar também que o presente estudo não utilizou-se do mesmo tipo de protocolo de fadiga, e não avaliou-se a fadiga com medição de marcadores bioquímicos. Embora sejam trabalho realizados em membros inferiores, eles não corroboram com os achados deste estudo.

Todavia, há estudos assim como o presente, cujos resultados também indicaram que a aplicação do laser após fadiga induzida não foi significante em comparação ao grupo que não foi irradiado. Higashi et al. ${ }^{19}$ aplicaram o LBP em mulheres jovens, e indicou-se que a laserterapia 
após o protocolo de exercício não aumentou o número de contrações musculares da reavaliação. Em outro estudo realizado por Toma et al. ${ }^{20}$, concluiu-se que a aplicação do LBP pós-fadiga não apresentou atraso dos índices de fadiga na eletromiografia de mulheres idosas. Por mais que tenha postulado-se em tal pesquisa que a laserterapia pode aumentar a oferta de energia muscular e culminar com a melhoria do desempenho do músculo aplicado retardando a situação da fadiga, o que observou-se nos resultados destes dois estudos é que o desempenho muscular não foi melhorado com a aplicação do laser, assim como no presente estudo.

A aplicação da LBP em alguns estudos não interferiu no desempenho muscular em homens sedentários relacionados ao desenvolvimento da fadiga muscular ${ }^{21}$ bem como na produção média de força durante a CIVM avaliada ${ }^{22}$.

Todavia, no estudo de Azevedo ${ }^{22}$ relatou-se mudança no padrão de recrutamento muscular para o grupo que foi irradiado, visto que foi mantido o mesmo nível de disparo de unidades motoras quando comparado ao momento antes da aplicação, ao contrário do grupo placebo, cujo recrutamento muscular foi menor. Trata-se de efeitos que, quando comparados a demais estudos, evidenciam a necessidade da observância da aplicação do LBP vinculada aos objetivos no atleta, e sua implicação efetiva ou não baseada nas evidências atuais sobre a laserterapia.

Deste modo, em meio aos estudos e pesquisas que divergem em suas conclusões, observa-se que o laser tem apresentado efeitos distintos no que se refere ao desempenho muscular de atletas. Novos estudos devem ser realizados com o intuito de conhecer o real efeito da LBP na fadiga muscular de atletas, e sugere-se que sejam verificados os efeitos crônicos desta modalidade, já que ainda estão escassos na literatura.

Além disto, deve-se considerar, quando da análise do efeito agudo, que o principal efeito a se observar seja a redução da amplitude da fadiga e não a melhora da desempenho de força, pois, de forma aguda, a LBP parece não ter o potencial de melhorar o desempenho muscular após a realização de protocolos de fadiga.

Aponta-se como limitações deste estudo a falta de avaliação por meio de marcadores bioquímicos indicadores da fadiga, a não observância do estado nutricional dos atletas, e o tipo de laser pontual utilizado, levando um tempo de aplicação alto, proporcionando um longo tempo de recuperação para os dois grupos antes da reavaliação do sinal eletromiográfico.

\section{CONCLUSÃO}

Após o protocolo de fadiga proposto, uma única aplicação de laser de baixa potência não foi suficiente para produzir efeitos positivos e negativos no desempenho de força e no sinal eletromiográfico do músculo bíceps braquial de atletas de voleibol.

Todos os autores declararam não haver qualquer potencial conflito de interesses referente a este artigo.

CONTRIBUIÇÕES DOS AUTORES: Cada autor contribuiu individual e significativamente para o desenvolvimento do manuscrito. JLF (0000-0003-3261-443X)*: foi a principal contribuinte para a redação do manuscrito, participou da coleta de dados aplicando o protocolo de fadiga e interpretação dos dados. ACMR (0000-0003- 23580514)*: participou do conceito intelectual, desenho do estudo e discussão dos resultados. EFBC (0000-0001-6901-9082)*: avaliou os dados, realizou a análise estatística e participou da interpretação dos resultados. FGLC (0000-0002-1915-2886)*: participou da coleta e analise dos dados relacionados a eletromiografia. RLAP (0000-0001-7790900X)* realizou as aplicações de laserterapia (ativo e placebo). MSK (0000-0002-0721-7582)*: participou do conceito intelectual do estudo e fez a revisão da versão final. CRP (0000-0001- 6907-3345)*: orientou o trabalho, participou do conceito intelectual do estudo e fez a revisão da versão final. * ORCID (Open Researcher and Contributor ID).

\section{REFERÊNCIAS}

1. Albuquerque DD, Pereira EN, Santos NGB, Brito LS, Oliveira AJJV. Assistência fisioterapêutica esportiva na prática do voleibol: um relato de extensão universitária. Revista Digital EFDeportes. 2014;18(188):1. Disponível em: http://www.efdeportes.com/efd188/assistencia-fisioterapeutica-na-pratica-do-voleibol.htm

2. Oliveira PR. Particularidades das açōes motoras e características metabólicas dos esforços específicos do voleibol juvenil e infanto-juvenil feminino. Rev Fac Claretianas. 1997;6:49-55.

3. Watkins J, Green BN. Volleyball injuries: a survey of injuries of ScottishNational League male players. Br J Sports Med. 1992;26(2):135-7.

4. Aagaard $H$, Scavenius $M$, Jørgensen U. An epidemiological analysis of the injury pattern in indoor and in beach volleyball. Int J Sports Med. 1997;18(3):217-21.

5. Gefen A, Megido-Ravid M, Itzchak Y, Arcan M. Analysis of muscular fatigue and foot stability during high-heeled gait. Gait Posture. 2002;15(1):56-63.

6. Leal Junior EC, Lopes-Martins RA, Vanin AA, Baroni BM, Grosselli D, De Marchi T, et al. Effect of $830 \mathrm{~nm}$ lowlevel laser therapy inexercise-induced skeletal muscle fatigue in humans. Lasers Med Sci. 2009;24(3):425-31.

7. Leal Junior EC, Lopes-Martins RA, Dalan F, Ferrari M, Sbabo FM, Generosi RA, et al. Effect of 655-nm low-level lasertherapy on exercise-induced skeletal muscle fatigue in humans. Photomed Laser Surg. 2008;26(5):419-24.

8. Silveira PC, Silva LA, Tuon T, Freitas TP, Streck EL, Pinho RA. Efeitos da laserterapia de baixa potência na reposta oxidativa epidérmica induzida pela cicatrização de feridas. Rev Bras Fisioter. 2009;13(4):281-7.

9. Silveira PC. Investigação do laser de baixa potência na recuperação de lesões epidérmicas e musculares em roedores [tesis]. Florianópolis: Universidade Federal de Santa Catarina (UFSC); 2013.

10. Ferraresi C, de Brito Oliveira T, de Oliveira Zafalon L, de Menezes Reiff RB, Baldissera V, de Andrade Perez $\mathrm{SE}$, et al. Matheucci oflow level laser therapy $(808 \mathrm{~nm})$ on physical strength training in humans. Lasers Med Sci. 2011;26(3):349-58.

11. Surface EMG for Non Invasive Assessment of Muscles (SENIAM). Recommendations for sensor locations in arm or hand muscles. [Internet]. [acesso em 2014 abr 25]. Disponivel em: <seniam.org/ bicepsbrachii.html>.
12. Ivey FM, Roth SM, Ferrell RE, Tracy BL, Lemmer JT, Hurlbut DE, et al. Effects of age,gender, and myostatin genotype on the hypertrophic response to heavy resistancestrength training. J Gerontol A Biol Sci Med Sci. 2000;55(11):M641-8.

13. Pasquet B, Carpentier A, Duchateau J, Hainaut K. Muscle fatigue during concentric and eccentric contractions. Muscle Nerve. 2000;23(11):1727-35.

14. Kawakami Y, Amemiya K, Kanehisa H, Ikegawa S, Fukunaga T. Fatigue responses of human triceps surae muscles during repetitive maximal isometric contractions. J Appl Physiol (1985). 2000;88(6):1969-75.

15. Gorelick M, Brown JM, Groeller H. Short-duration fatigue alters neuromuscular coordination of trunk musculature: implications for injury. Appl Ergon. 2003;34(4):317-25.

16. Gandevia SC. Neural control in human muscle fatigue: changes in muscle afferents, motoneurones and motor cortical drive [corrected]. Acta Physiol Scand. 1998 Mar;162(3):275-83. Review. Erratum in: Acta Physiol Scand 1998;163(3):305

17. Santos S, Piucco T, Cunha-dos-Reis D. Fatores que interferem nas lesöes de atletas amadores de voleibol. Rev Bras Cineantropom Desempenho Hum. 2007;9(2)189-95.

18. Reis FA. Efeitos da aplicação do laser de baixa intensidade (830nm) no desempenho muscular antes e após protocolo de fadiga induzida pelo exercício em atletas de futebol [tese]. Campo Grande: Universidade Federal de Mato Grosso do Sul (UFMS): 2013.

19. Higashi RH, Toma RL, Tucci HT, Pedroni CR, Ferreira PD, Baldini G, et al. Effects of low-level laser therapy on biceps braquialis muscle fatigue in young women. Photomed Laser Surg. 2013;31(12):586-94

20. Toma RL, Tucci HT, Antunes HK, Pedroni CR, de Oliveira AS, Buck I, et al. Effect of $808 \mathrm{~nm}$ low-level laser therapy in exercise-induced skeletal muscle fatigue in elderly women. Lasers Med Sci. 2013;28(5):1375-82

21. Leal-Junior ECP, Nassar FR, Tomazoni SS, Bjordal JM, Lopes-Martins RAB. A laserterapia de baixa potência melhora o desempenho muscular mensurado por dinamometria isocinética em humanos. Fisioter Pesquisa. 2010;17(4):317-21

22. Azevedo RC. Laserterapia de baixa intensidade e recuperação muscular em fadiga: Ensaio clínico controlado. Perspect online: Biol Saúde. 2011;3(1):23-30. 